\title{
TRATAMENTO CONSERVADOR DOS HEMATOMAS EXTRADURAIS LAMINARES NA INFÂNCIA
}

\author{
ORILDO CIQUINI JUNIOR * - ALBERTO L. MOURA DOS SANTOS ** \\ LUIZ A. MAN REZA *** - J. PÍNDARO P. PLESE **** \\ RAUL MARINO JÚNIOR *****
}

\begin{abstract}
RESUMO - Os autores relatam sua experiência com o tratamento conservador de 15 crianças com hematoma extradural (HEI» laminar traumático. Todas, por ocasião do diagnóstico, apresentavam-se neurologicamente estáveis e sem sinais de herniação intracraniana. Estes HED foram descobertos em períodos de 30 minutos e 5 dias depois do trauma, a maioria estando localizados na região parietal. $O$ HED foi reabsorvido* espontaneamente em todos os casos, num período que variou de 2 a 12 dias. Os critérios para seleção destes pacientes e os cuidados com essa modalidade de tratamento do HED são discutidos.

PALAVRAS-CHAVE: hematoma extradural em crianças, tratamento conservador, tomografia computadorizada.

Nonsurgical management of laminar extradural hematomas in children

SUMMARY - We sucessfully treated by nonsurgical methods 15 children with laminar epidural hematomas $(E H)$, with minimal neurological symptoms and no signs of brain herniation. These EH were discovered 30 minutes to 5 days after heiad injury. The majority were localized in the parietal region. All children recovered without surgery from 2 to 12 days after hospitalization and all had evidence on CT scan of spontaneous clot reabsortion. We discuss the criteria for patient selection for this kind of treatment on EH in children.

KEY WORDS: extradural hematoma in children, conservative management, computerized tomography.
\end{abstract}

A maioria dos neurocirurgiões é de opinião que o tratamento dos hematomas extradurais traumáticos (HED) na infância é cirúrgico 1-3,8,9. Genarelli e col.6 enfatizam que a mortalidade e morbidade desta patologia está diretamente relacionada ao quadro neurológico do paciente no momento da cirurgia. Choux e col.3 afirmam que o número de HED assintomáticos, nessa faixa etária, é pequeno e que essas crianças podem apresentar súbita piora neurológica; são enfáticos em afirmar que seu tratamento é cirúrgico, devendo ser realizado o mais precocemente possível.

Com o advento da tomografia computadorizada encefálica (TC), alguns autores aconselham observação neurológica rigorosa e repetida, acompanhada de TC seriadas, em pacientes que apresentam HED laminares quando da admissão hospitalar, mas com quadro neurológico estável 2,5,7,8,11-13. Aventam a hipótese de que esses hematomas laminares são reabsorvidos de maneira semelhante aos subdurais, não merecendo tratamento cirúrgico 8,12.

O intuito deste estudo é apresentar os resultados obtidos com o tratamento conservador de 15 crianças com HED laminar.

Estudo realizado na Disciplina de Neurocirurgia do Departamento de Neurologia da Faculdade de Medicina da Universidade de São Paulo (FMUSP): * Neurocirurgião; ** Residente de Neurocirurgia; $* * *$ Professor Assistente; $* \star * *$ Professor Associado; $* \star \star \star *$ Professor Tituliar.

Dr. José Píndaro Pereira Plese - Clínica Neurológica, Hospital das Clinicas, FMUSP - Caixa Postal 8091 - 01065 São Paulo SP - Brasil. 


\section{CASUÍSTICA}

Durante os anos de 1987 a 1981 foram admitidas, no Pronto Socorro de Neurocirurgia do Hospital das Clínicas da FMUSP, 55 crianças com diagnóstico de HED confirmado mediante TC. Destas, 15 foram tratadas conservadoramente, pois os hematomas eram laminares e não acarretavam aumento da pressão intracraniana (PIC) ou sinais neurológicos fecais decorrentes de hérnias cerebrais. Dez eram do sexo feminino e 5 , do masculino. A idade variou de 11 meses a 12 anos, com média de 6,25 anos. Deve-se ressaltar que 12 crianças tinham idade superior a 3 anos.

A análise da Tabela 1 mostra que queda de altura superior a 2 metros e atropelamento foram os tipos de acidentes mais frequentes $(53,4 \%)$, seguindo-se queda de altura superior a 0,5 metro $(20 \%)$ e queda de escada $(13,3 \%)$. Acidentes em que objetos se chocaram centra o crânio corresponderam a $13,3 \%$ dos casos. Pela Tabela 2 evidencia-se que $80 \%$ das crianças foram atendidas no hospital nas primeiras 24 horas após o acidente. Destas, $40 \%$ nas 12 horas subsequentes. Tiveram sua admissão, após 24 horas, 3 pacientes (20\%). De maneira genérica esse tempo variou de 30 minutos a 5 dias após o traumatismo craniano (TCE).

Na Tabela 3 mostra-se que, por ocasião da chegada ao Pronto Socorro, sonolência (38,9\%), vômitos $(33,4 \%)$ e cefaléia $(22,2 \%)$ foram os principais sinais e sintomas apresentados pelos pacientes. Deve-se ressaltar que uma criança $(5,5 \%)$ estava assintomática. Quanto ao exame neurológico (Tabela 4), 7 crianças apresentavam escores na escala de coma de Glasgow (SCG) de $15(53,4 \%), 2$ entre $12-14(15,4 \%)$ e 3 , de $10(23,2 \%)$. Dos três pacientes com idade inferior a 3 anos, em que a SCG não se aplica, 2 estavam sonolentos e sem sinais focais e 1, assintomático. Nos pacientes com escore inferior a 14 na SCG, o quadro neurológico não foi considerado como consequente ao HED.

O estudo radiológico simples de crânio (Tabela 5), realizado em todos os pacientes, mostrou sinais de fratura em $11(73,4 \%)$, sendo normal em $4(26,6 \%)$. A TC, realizada em todos os pacientes, indicou a presença de HED (Fig. 1); um paciente apresentava sinais tomográficos de contusão fronto-basal esquerda (SCG 12) e outro, «swelling» hemisférico (SCG 10).

Quanto à localização do HED $^{1}$, na Tabela 6 mostra-se que 7 (46,7\%) eram parietais, 3 eram parieto-oecipitais $(20 \%)$ e 2 , occipitais $(13,3 \%)$; localizações na região frontal $(6,6 \%)$ temporal $(6,6 \%)$ e na fossa posterior $(6,6 \%)$ foram menos frequentes.

Todas as crianças permaneceram internadas sob rigorosa observação neurológica, sendo submetidas a TC seriadas. Em todas, houve evidência tomográfica de absorção do HED. Os pacientes tiveram alta hospitalar com escore, na SCG, de 15; em nenhum houve intervenção cirúrgica. O período de internação dessas crianças variou dei 2 a 12 dias, com média de 3,73 dias. A permanência no hospital foi de 2 dias em $60 \%$ dos casos, de 2 a 5 dias em $26,7 \%$ e superior a 5 dias em $13,3 \%$ (Tabela 7 ).

Qaunto ao número de TC seriadas, 12 crianças $(80 \%)$ necessitaram de um exame tomográfico para o controle do HED. Em 3 pacientes mais de uma TC foi necessária para comprovar a absorção do HED (Fig. 2).

Tabela 1. Tipo de acidente.

Acidente No de casos

\begin{tabular}{lll}
\hline \hline Queda de altura superior a 2 metros & 4 & $(26,7 \%)$ \\
Atropelamento & 4 & $(26,7 \%)$ \\
Queda de altura superior a 0,5 metro & 3 & $(20,0 \%)$ \\
Queda da escada & 2 & $(13,3 \%)$ \\
Outros & 2 & $(13,3 \%)$ \\
\hline
\end{tabular}


Tabela 2. Espaço de tempo acidente/admissão hospitalar (30 minutos a 5 dias).

\begin{tabular}{ccc}
\hline Tempo & No de casos \\
\hline$<6$ horas & 6 & $(40,0 \%)$ \\
$6-24$ horas & 6 & $(40,0 \%)$ \\
$>24$ horas & 3 & $(20,0 \%)$ \\
\hline
\end{tabular}

Tabela 3. Sinais e sintomas à admissão hospitalar.

\begin{tabular}{lcc}
\hline Sinais e sintomas & No de casos \\
\hline \hline Sonolência & 7 & $(38,9 \%)$ \\
Vômitos & 6 & $(33,4 \%)$ \\
Cefaléia & 4 & $(22,2 \%)$ \\
Assintomático & 1 & $(5,5 \%)$
\end{tabular}

Tabela 4. Exame neurológico à admissão hospitalar.

\begin{tabular}{lll}
\hline Exame & \multicolumn{2}{c}{ No de casos } \\
\hline SCG 15 & 7 & $(53,4 \%)$ \\
SCG $12-14$ & 2 & $(15,4 \%)$ \\
SCG 10 & 3 & $(23,2 \%)$
\end{tabular}

SCG, escala de coma de Glasgow.
Tabela 5. Radiografia simples de crânio.

\begin{tabular}{lrr}
\hline Achado & No de casos \\
\hline $\begin{array}{l}\text { Sinais de fratura } \\
\text { Ausência de sinais de fratura }\end{array}$ & $\begin{array}{rrr}(73,4 \%) \\
(26,6 \%)\end{array}$ \\
\hline
\end{tabular}

Tabela 6. Localização do hematoma.

\begin{tabular}{lcc}
\hline Topografia & No de casos \\
\hline Parietal & 7 & $(46,7 \%)$ \\
Parieto-occipital & 3 & $(20,0 \%)$ \\
Occipital & 2 & $(13,3 \%)$ \\
Outros & 3 & $(20,0 \%)$
\end{tabular}

Tabela 7. Período de internação.

\begin{tabular}{lrl}
\hline \multicolumn{1}{c}{ Periodo } & No de casos \\
\hline 2 dias & 9 & $(60,0 \%)$ \\
$2-5$ dias & 4 & $(26,7 \%)$ \\
maior que 5 dias & 2 & $(13,3 \%)$ \\
\hline
\end{tabular}

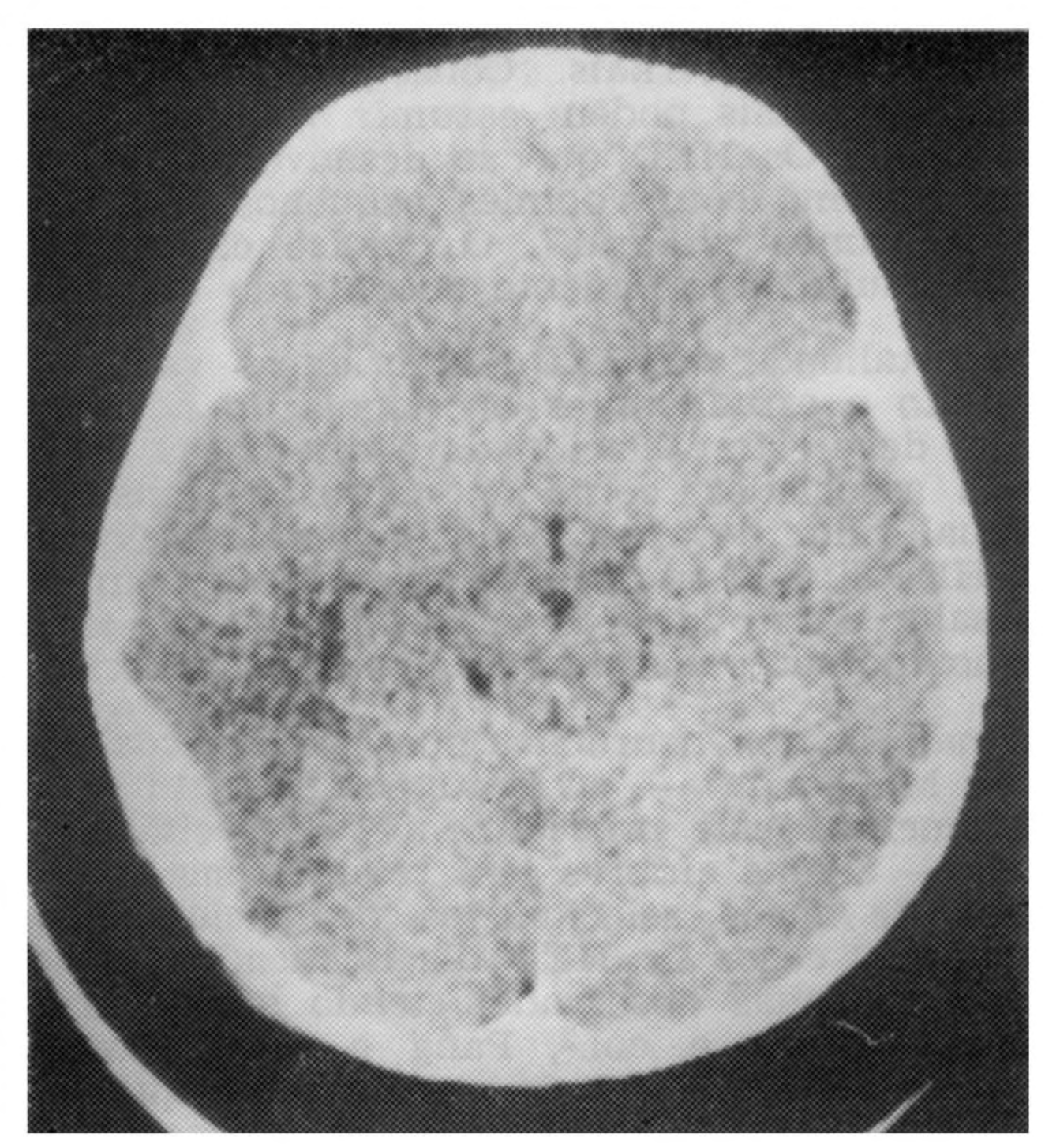

Fig. 1. TC mostrando HED laminar parietal esquerdo. Não se observam sinais de herniações intracranianas. 

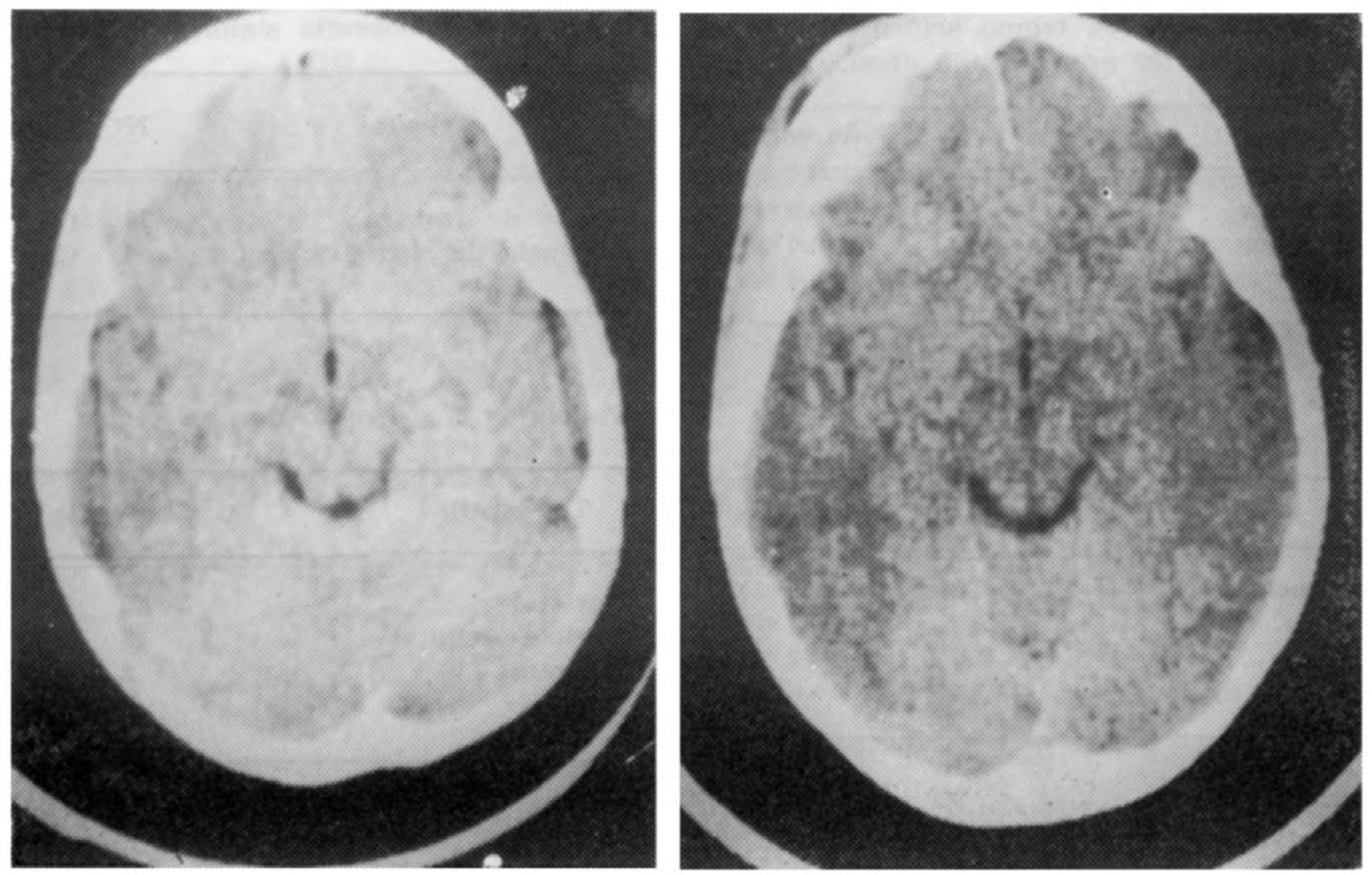

Fig. 2. A esquerda, TC mostrando HED laminar frontal esquerdo. A direita, TC realizada 4 dias apos, mostrando regressão parcial espontanea do HED.

\section{COMENTÁRIOS}

Os HED, embora raros, constituem importante complicação dos traumatismos cranianos na infância. Sua incidência é de 2 a $3 \%$ quando se analisam as maiores casuísticas L3.9. Os lactentes toleram melhor os aumentos da PIC em virtude de apresentarem as suturas cranianas ainda abertas, fontanelas e amplos espaços celulares e cisternais. Consequentemente, os HED localizados nas regiões frontais e parietais podem assumir volumes consideráveis por ocasião do seu diagnóstico i. Os HED que se desenvolvem na região subtemporal e na fossa posterior, mesmo com volumes pequenos, podem acarretar precocemente compressão do tronco cerebral 9. O quadro clínico clássico de intervalo lúcido, observado no adulto, é raramente encontrado em crianças ${ }^{3}$.

$O$ índice de mortalidade do HED em crianças varia de 0 a $17 \%$, dependendo de ter sido feito o estudo antes ou depois do advento da $\mathrm{TC}^{1}-^{3} \wedge$. Essa discrepância aparente deve estar relacionada a diferenças na incidência de lesões intraparenquimatosas associadas, como as contusões encefálicas, relatadas em algumas séries ${ }^{3}$. Igual valor deve ser dado ao tempo decorrido entre o trauma e o diagnóstico do HED e a instituição do tratamento neurocirúrgico ${ }^{4}$ A maioria dos autores ressalta o fato de que há nítida relação entre o estado neurológico do paciente no momento da cirurgia e seu prognósticó。

Apesar de a maioria dos neurocirurgiões pediátricos aceitarem como verdadeiro o axioma de McLaurin» - «O tratamento definitivo do HED é sempre cirúrgico e o retardamento deste procedimento é inaceitável após se ter estabelecido o diagnóstico» - alguns autores têm relatado sua experiência, com o tratamento conservador de casos selecionados de paciente com HED 2,5,7,8,10-15. Weaner e col. ${ }^{16}$ relatam 2 casos de HED diagnosticados 16 horas e 3 dias após o TCE, os quais foram apenas observados, tendo havido reabsorção dos hematomas. Recentemente, Bullock e col.2, Pang e col. ${ }^{12}$, Pozzati e Tognetti ${ }^{13}$ e Knuckey e col. ${ }^{8}$ relatam série maiores de pacientes em que eSse tipo de conduta foi instituída, com excelente evolução clínica. A análise de algumas casuísticas mostra que $3,6 \%$ a $64,9 \%$ dos pacientes com HED, tratados conservadoramente, necessitaram de intervenção neurocirúrgica para drenagem do hematoma, devido a aumento volumétrico dele associado a deterioração do quadro neurológico do 
paciente ${ }^{8} \gg^{14}-{ }^{15}$. Em nossa série nenhum paciente necessitou de tratamento neurocirúrgico.

O tempo decorrido entre o TCE e o diagnóstico de HED é importante, como fator prognóstico, na piora neurológica. Knuckey e col. ${ }^{8}$ afirmam que HED pequenos, diagnosticados 6 horas após o TCE, têm $13 \%$ de chance de apresentar aumento volumétrico e requererem tratamento cirúrgico. Nos HED diagnosticados precocemente, nas primeiras 6 horas, esse índice sobe para $43 \%$. Afirmam ainda que os HED pequenos, diagnosticados após 6 horas do TCE, podem ser tratados conservadoramente com margem de segurança grande. Recomendam repetir a TC, após 6 horas do TCE nos pacientes neurologicamente estáveis, cuja TC foi realizada precocemente.

Frankhauser e col. ${ }^{5}$ e Smith e Miller ${ }^{15}$ destacam a importância do momento da realização da TC após o TCE. Relatam a verificação de pacientes que apresentavam TC normal após o acidente e que vieram a desenvolver HED tardiamente, necessitando de tratamento cirúrgico. Isso vem contra a opinião de McLaurim e Towbin9, de que o HED atinge o seu tamanho máximo em breve período de tempo após o TCE. A análise da literatura mostra que, na maioria dos pacientes com HED pequenos e tratados conservadoramente, o diagnóstico foi feito entre o $V$ e o $17^{9}$ dias pós-TCE ${ }^{8}$, Pozzati e Tognetti ${ }^{13}$, Sakai e col. ${ }^{14}$ e Knuckey e col. ${ }^{8}$ relatam casos em que o diagnóstico foi firmado nas primeiras 24 horas e resultados ótimos foram obtidos com essa modalidade de tratamento. Em nossa série, em $80 \%$ dos casos o diagnóstico de HED foi feito neste período de tempo.

Alguns autores relacionam a presença de fraturas da abóbada craniana, que cruzam seios venosos ou o trajeto de artérias meníngeas, com HED laminares, dado sugestivo da necessidade de tratamento cirúrgico ${ }^{1}{ }^{3} \wedge$. Eles enfatizam que poderá haver descompensação tardia por aumento do sangramento. Em nossa série» $\mathbf{7 3 . 4 \%}$ apresentavam fraturas cranianas e não houve relação com aumento volumétrico do HED.

Alguns autores criticam essa modalidade de tratamento dos HED, devido ao maior período de internação e tomografias computadorizadas encefálicas seriadas, o que aumentaria o custo do tratamento ${ }^{13},{ }^{14}$. Contrariando a opinião desses autores, nossa casuística mostrou que 86,7\% dos pacientes permaneceram internados durante 2 a 5 dias.

Em conclusão, em crianças oligossintomáticas, com hematomas extradurais laminares sem efeito de massa, o tratamento pode ser conservador. Quando o diagnóstico for feito antes de 6 horas após o TCE, nova TC deverá ser realizada dentro das 18 horas subsequentes. Tomografias seriadas devem ser feitas diante de piora do estado neurológico, com a finalidade de avaliar o volume do hematoma.

\section{REFERENCIAS}

1. Ammirati M, Tomita T. Epidural hematomas in infancy and childhood. Riv Neurosc Ped ( $J$ Ped Neurose) 1985, 1:123-128.

2. Bullock R, Smith RM, VanDellen J. Nonoperative management of extradural hematoma. Neurosurgery 1985, 16:602-606.

3. Choux M, Lena G, Genitore L, Empieme E, Dechambendis G. Pediatric head injuries. In Braáïman $R$ (eds): Handbook of Clinical Neurology, Vol 13(57), Head Injury. Amsterdam: Elsevier, 1990, p 327-343.

4. Ericson $\mathbf{K}$, Häransson $\mathbf{S}$. Computed tomography of epidural hematomas: association with intracranial lesions and clinical correlation. Acta Radiol 1981, 22:513-519.

5. Frankhauser H, Uske A, de Tribolet $N$. Delayed epidural hematoma: a propos of a serie of 8 cases. Neurochirurgie 1983, 29:255-260.

6. Gennarelli TA, Spilman GM, Langfih TW, Gildenberg PL, Harington T, Jane JA, Marshall LF, Miller JD, Pitts LH. Influence of the type of intracranial lesion on outcome from severe head injury: a multicenter study using a new classification system. $J$ Neurosurg 1982, 56:26-32.

7. Illingworth $R$, Schawdow $H$. Conservative management of intracranial extradural haematomas presenting late. J Neurol Neurosurg Psychiatry 1983, 46:558-560. 
8. Knuckey NW, Gelbard S, Epstein MH. The management of asymptomatic epidural hematomas. J Neurosurg 1989, 70:392-396.

9. McLaurin RL, T'owbin RB. Post-traumatic hematomas. In McLaurin RL, Schult L, Veres JL, Epstein F (eds): Pediatric Neurosurgery Ed 2. Philadelphia: Saunders, 1989, 277-289.

10. Mendelow AD, Karmini Z, Paul KS. Extradural haematoma effect of delayed treatment. Br Med J 1979, 1:1240-1242.

11. Negishi H, Lee Y, loth K, Suzuki J, Nishino M, Takada S, Yamasaki S. Monsurgical management of epidural hematoma in neonates. Pediatr Neurol 1989, 5:253-256.

12. Pang D, Horton JA, Herron JM, Wilberg SE, Vries JK. Nonsurgical management of extradural hematomas in children. J Neurosurg 1983, 59:958-971.

13. Pozzati E, T'ognetti F. Spontaneous healing of acute extradural hematomas: study of twenty-two cases. Neurosurgery 1986, 18:696-700.

14. Sakai H, Taragi H, Ohtiaka H. Serial changes in acute extradural hematoma size and associated changes in level of consciousness and intracranial pressure. $J$ Neurosurgery 1988, 68:566-570.

15. Smith HK, Miller JD. The danger of an ultra-early computed tomographic scan in a patient with an evolving acute epidural hematoma. Neurosurgery 1991, 29:258-260.

16 "Weaver D, Pobereskin L, Jane JA. Spontaneous resolution of epidural hematomas: report of two cases. J Neurosurg 1981, 54:248-251. 